\title{
Review of T-2307, an Investigational Agent That Causes Collapse of Fungal Mitochondrial Membrane Potential
}

\author{
Nathan P. Wiederhold
}

Citation: Wiederhold, N.P. Review of T-2307, an Investigational Agent That Causes Collapse of Fungal Mitochondrial Membrane Potential. J. Fungi 2021, 7, 130. https://doi.org/10.3390/ jof7020130

Academic Editor: Richard Calderone

Received: 20 January 2021

Accepted: 8 February 2021

Published: 11 February 2021

Publisher's Note: MDPI stays neutral with regard to jurisdictional claims in published maps and institutional affiliations.

Copyright: (c) 2021 by the author Licensee MDPI, Basel, Switzerland. This article is an open access article distributed under the terms and conditions of the Creative Commons Attribution (CC BY) license (https:// creativecommons.org/licenses/by/ $4.0 /)$.
Fungus Testing Laboratory, Department of Pathology and Laboratory Medicine, University of Texas Health Science Center, San Antonio, TX 78229, USA; wiederholdn@uthscsa.edu

\begin{abstract}
Invasive infections caused by Candida that are resistant to clinically available antifungals are of increasing concern. Increasing rates of fluconazole resistance in non-albicans Candida species have been documented in multiple countries on several continents. This situation has been further exacerbated over the last several years by Candida auris, as isolates of this emerging pathogen that are often resistant to multiple antifungals. T-2307 is an aromatic diamidine currently in development for the treatment of invasive fungal infections. This agent has been shown to selectively cause the collapse of the mitochondrial membrane potential in yeasts when compared to mammalian cells. In vitro activity has been demonstrated against Candida species, including C. albicans, C. glabrata, and C. auris strains, which are resistant to azole and echinocandin antifungals. Activity has also been reported against Cryptococcus species, and this has translated into in vivo efficacy in experimental models of invasive candidiasis and cryptococcosis. However, little is known regarding the clinical efficacy and safety of this agent, as published data from studies involving humans are not currently available.
\end{abstract}

Keywords: T-2307; aromatic diamidine; in vitro susceptibility; mitochondrial membrane; mitochondrial membrane potential; Candida; Cryptococcus

\section{Introduction}

Infections caused by fungi are of increasing clinical concern. These include invasive diseases caused by strains of Candida species that are resistant to clinically available antifungals. As highlighted by the World Health Organization global surveillance report on antimicrobial resistance, resistance in non-albicans Candida species to fluconazole has been reported at rates of $30 \%$ or higher in multiple countries on several continents [1]. At some centers in the United States, increased rates of nonsusceptibility to the echinocandins have also been reported in C. glabrata [2,3]. Interestingly, some studies have also noted coresistance to both the echinocandins and the azoles in C. glabrata isolates [3,4]. Recently, the problem of antifungal resistance has been highlighted by the emergence of Candida auris, as many strains of this clinically significant pathogen are multidrug-resistant [5], and pan-antifungal resistance has also been reported [6,7]. In addition, the utility of clinically available antifungals is often limited by drug-drug interactions and toxicities, which can occur frequently in patients at a high risk of invasive fungal infections who are often receiving multiple concomitant medications [8-11]. Thus, there is a true need for the development of new antifungals.

T-2307 (4-\{3-[1-(3-\{4-\{amino(imino)methyl]phenoxy\}propyl)piperidin-4-yl]propoxy\} benzmide) is an investigational agent currently under evaluation and in development for the treatment of invasive fungal infections, including those caused by Candida species that are resistant to clinically approved antifungals, including azole- and echinocandin-resistant strains. The initial preclinical and early-stage clinical development of T-2307 has been conducted by FUJIFILM Toyama Chemical Co., Ltd., (Tokyo, Japan), which has recently assigned the rights for developing and marketing outside of Japan to Appili Therapeutics (Halifax, NS, Canada; ATI-2307) (http:/ /fftc.fujifilm.co.jp/en/news/news191121e.html, 
accessed on 15 January, 2021). This review discusses the mechanism of action of T-2307, its spectrum of activity against various fungi, and what is known about its effectiveness in experimental models of invasive fungal infections. Key features of T-2307 are shown in Table 1.

Table 1. Key features of T-2307.

\begin{tabular}{cc} 
Mechanism of Action & $\begin{array}{c}\text { Causes the collapse of the mitochondrial membrane potential by targeting respiratory } \\
\text { chain enzymatic complexes III and IV in the inner mitochondrial membrane of yeasts. }\end{array}$ \\
Spectrum of Activity & $\begin{array}{r}\text { Candida species, including azole- and echinocandin-resistant isolates, } \\
\text { Cryptococcus neoformans \& C. gattii. } \\
\text { Variable activity against Aspergillus species. }\end{array}$ \\
In vivo activity & $\begin{array}{r}\text { Limited in vitro data against fungi suggests limited to no activity against } \\
\text { Fusarium, Scedosporium, and the Mucorales. }\end{array}$ \\
\hline Pharmacokinetic and clinical data & $\begin{array}{r}\text { Efficacy in murine models of invasive candidiasis caused by C. albicans, C. glabrata, } \\
\text { and C. auris; reductions in the ocular fungal burden caused by C. albicans. } \\
\text { Efficacy in murine models of systemic aspergillosis due to Aspergillus fumigatus and } \\
\text { cryptococcosis caused by C. neoformans and C. gattii. }\end{array}$ \\
\hline
\end{tabular}

\section{Structure and Mechanism of Action}

Structurally, T-2307 is an aromatic diamidine, similar to pentamidine and furamidine (DB75) (Figure 1) [12]. Pentamidine can be used to treat pneumocystis, leishmaniais, and trypanosomiasis, and it is known that pentamidine and furamidine cause the collapse of the mitochondrial membrane potential within Saccharomyces cerevisiae [13-15]. A similar mechanism of action was suspected for T-2307 when it was observed that the degree of its activity against Candida glabrata was strongly influenced by the type and concentration of the carbon source in the growth medium. The trailing effect (i.e., incomplete inhibition of growth at concentrations above the minimum inhibitory concentration [MIC]) was reduced at lower glucose concentrations and was absent when the nonfermentative carbon source glycerol was used [14]. Similarly, S. cerevisiae cells grown in medium containing glycerol were also more sensitive to the effects of pentamidine and furamidine when compared to when they were grown in the presence of glucose. Similar results have also been reported for both T-2307 and pentamidine against C. albicans and S. cerevisiae when growth inhibition was compared between media containing dextrose and glycerol [13]. Interestingly, both the fermentative (in the presence of dextrose) and nonfermentative (in the presence of glycerol) growth of Cryptococcus neoformans was similarly inhibited by T-2307.

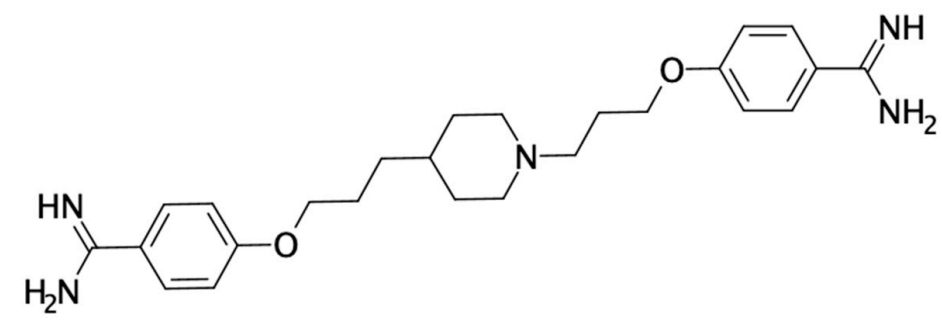

(A)<smiles>N=C(N)c1ccc(OCCCCCOc2ccc(C(=N)N)cc2)cc1</smiles>

(B)

Figure 1. Chemical structures of the aromatic diamidines (A) T-2307 and (B) pentamidine. 
In order to assess the mechanism of action of T-2307 against yeast, Shibata et al. conducted a series of experiments [13]. To investigate the effects of T-2307 on the mitochondrial function in S. cerevisiae and C. albicans, the accumulation of the fluorescent dye MitoTracker Red CMXRos (MTR), a mitochondrial marker that concentrates in active mitochondria by membrane potential, was evaluated by microscopy. The presence of T-2307 at a concentration of $1 \mu \mathrm{M}$ resulted in a less intense staining of mitochondria by MTR, indicating that the mitochondrial function had collapsed. These results were similar to those observed with carbonyl cyanide $m$-chlorophyenylhydrazone (CCCP), a protonophore and uncoupler of oxidative phosphorylation. In both S. cerevisiae and C. albicans, the number of whole cells with intense staining of the mitochondria decreased in the presence of T-2307 in a concentration-dependent fashion. In addition, a gradual and dose-dependent collapse of the mitochondrial membrane potential was also noted in mitochondria isolated from S. cerevisiae that were exposed to T-2307 or pentamidine. However, the T-2307 concentration required to cause a collapse in the membrane potential of at least $50 \%(30 \mu \mathrm{M})$ was markedly higher than the concentration required to inhibit the growth of the isolate (MIC $0.002 \mu \mathrm{M}$, or $0.001 \mu \mathrm{g} / \mathrm{mL}$ ). With pentamidine, a reduction in the mitochondrial membrane potential of at least $50 \%$ occurred at a concentration of $5 \mu \mathrm{M}$, while the MIC against $S$. cerevisiae was reported to be $1.5 \mu \mathrm{M}$, or $0.5 \mu \mathrm{g} / \mathrm{mL}$. The disconnect between the T-2307 concentration required to cause the collapse of the mitochondrial membrane potential and that needed to inhibit the growth of the organism (MIC) was postulated to be due to the intracellular accumulation of T-2307, as others have reported that this agent concentrates approximately 5000-fold within C. albicans cells from an extracellular medium via transporter mediated systems [16]. Subsequent work has indicated that T-2307 transport into C. albicans occurs through a high-affinity spermine and spermidine transport system that is regulated by Agp2, a plasma membrane protein involved in the update of L-carnitine and polyamines and other substrates in S. cerevisiae [17]. It is known that the uptake of polyamines, organic compounds having more than two amino groups, is dependent upon the mitochondrial membrane potential [18]. Interestingly, the activity of T-2307 against a S. cerevisiae strain with a $\triangle a g p 2$ mutation was markedly reduced when compared to the wild-type strain (MIC $>8 \mu \mathrm{g} / \mathrm{mL}$ vs. $0.00012 \mu \mathrm{g} / \mathrm{mL}$, respectively). The implications of this finding for the potential development of resistance to T-2307 are unknown.

The effect of T-2307 against rat liver mitochondria was also assessed [13]. No inhibitory effect on the rat mitochondrial membrane potential was observed with T-2307 at concentrations as high as $10 \mathrm{mM}$, which was at least 10 times higher than that observed with pentamidine, where collapse was observed at a concentration of $1 \mathrm{mM}$. Furthermore, the concentration of T-2307 within C. albicans cells was significantly higher than that observed in rat hepatocytes. Thus, T-2307 appears to have a greater selectivity for fungal rather than mammalian mitochondria and also concentrates to a greater degree within yeast cells when compared to mammalian cells.

To further assess the mechanism of action of T-2307 on the mitochondrial function, Yamashita et al. measured oxygen consumption in whole yeast cells [19]. Respiration was inhibited by T-2307 in a dose-dependent fashion, while respiration was not stimulated at any tested concentration (up to $1000 \mu \mathrm{M}$ ). Against intact yeast mitochondria, T-2307 caused a dose-dependent decrease in the rate of mitochondrial oxygen consumption in both the presence and absence of adenosine diphosphate (ADP). Similarly, potassium cyanide, a respiratory chain inhibitor, completely inhibited respiration under both basal and ADP-stimulated conditions, while oligomycin A, an ATP synthase inhibitor, did not affect the oxygen consumption rate in the absence of ADP. Thus, the oxygen consumption inhibition pattern observed with T-2307 indicates that this investigational agent inhibits the yeast mitochondrial respiratory chain. Further work from this study suggested that T-2307 induced mitochondrial dysfunction in yeast mainly through the inhibition of the respiratory chain enzyme complexes III and IV, and that ATP production was suppressed in a dose-dependent fashion, with the intracellular levels significantly decreased at T-2307 concentrations of $\geq 0.01 \mu \mathrm{M}$. However, against mitochondria isolated from a bovine heart, 
the T-2307 IC50 values were $>3000 \mu \mathrm{M}$ against all mitochondrial respiratory chain enzyme complexes. These results further demonstrate the selectivity of T-2307 against the yeast mitochondrial function.

\section{In Vitro Spectrum of Activity}

T-2307 has demonstrated an antifungal activity against several yeast species and some filamentous fungi, including isolates that are resistant to clinically approved azoles (Table 2). Against yeasts, it shows activity against Candida and Cryptococcus species, as well as Malassezia furfur. In an early screen for activity that included several ATCC and other reference strains of Candida and other yeasts and that utilized the Clinical and Laboratory Standards Institute (CLSI) broth microdilution methods with a prominent reduction in growth as the MIC endpoint, T-2307 demonstrated MICs ranging from $0.00025 \mu \mathrm{g} / \mathrm{mL}$ to $0.0039 \mu \mathrm{g} / \mathrm{mL}$ against Candida species, including four isolates of $C$. albicans, and single strains of C. dublieniensis, C. glabrata, C. guilliermondii, C. krusei, C. parapsilosis, and C. tropicalis each [12]. Against three C. neoformans isolates, the T-2307 MICs ranged from 0.0039 to $0.0078 \mu \mathrm{g} / \mathrm{mL}$, and against a single isolate of $M$. furfur the MIC was $0.0313 \mu \mathrm{g} / \mathrm{mL}$. Minimum fungicidal concentrations (MFC) were also determined, and the results were highly variable, with MFC values ranging from $0.0156 \mu \mathrm{g} / \mathrm{mL}$ to $0.0625 \mu \mathrm{g} / \mathrm{mL}$ against $C$. neoformans, but from $0.002 \mu \mathrm{g} / \mathrm{mL}$ to $>64 \mu \mathrm{g} / \mathrm{mL}$ against different Candida species. Little to no activity was reported against the single isolates of Saccharomyces cerevisiae and Trichosporon asahii that were included in this study (MIC and MFC values of $\geq 64 \mu \mathrm{g} / \mathrm{mL}$ ). Additional in vitro studies have reported that T-2307 maintains activity against echinocandin-resistant strains of both C. albicans and C. glabrata, with similar geometric mean (GM) MIC values reported for caspofungin-susceptible and -resistant isolates (T-2307 GM MIC range of $0.008 \mu \mathrm{g} / \mathrm{mL}$ to $0.0135 \mu \mathrm{g} / \mathrm{mL}$ against all studied isolates) [20,21]. Interestingly, a complete inhibition of growth was not achieved against C. albicans or C. glabrata isolates. Recently, in vitro activity has also been demonstrated against the emerging pathogen $C$. auris. In a study that used 23 C. auris isolates, including those available from the CDC FDA AR Bank (https:/ / www.cdc.gov/drugresistance/ resistance-bank/index.html, accessed on 15 January, 2021) and additional clinical isolates, the MICs for T-2307 ranged from $\leq 0.008 \mu \mathrm{g} / \mathrm{mL}$ to $0.015 \mu \mathrm{g} / \mathrm{mL}$, and the activity of this investigational agent was maintained against strains that were resistant to fluconazole [22]. A separate study also reported activity against 15 clinical isolates of $C$. gattii, with MICs ranging from $0.0078 \mu \mathrm{g} / \mathrm{mL}$ to $0.0625 \mu \mathrm{g} / \mathrm{mL}$ [23]. In a time-kill analysis also performed as part of this work, T-2307 demonstrated a concentration-dependent fungicidal activity with reductions in colony-forming units of $>3 \log _{10}$ at concentrations four times as high as the MIC value or higher. A similar fungicidal activity was also noted with amphotericin B.

Table 2. In vitro activity of T-2307. The results for which data are available for $>10$ isolates are shown. The endpoint that was used in all studies was a prominent inhibition of growth, and MICs are in $\mu \mathrm{g} / \mathrm{mL}$. MIC50 and MIC90 values represent MIC values at which 50\% and 90\% of the tested isolates were, respectively, inhibited.

\begin{tabular}{lccc}
\hline Species & MIC Range & MIC50 & MIC90 \\
\hline Candida albicans & $0.00025-0.008$ & $\leq 0.008$ & $\leq 0.008$ \\
Candida auris & $\leq 0.008-0.015$ & 0.015 & 0.015 \\
Candida glabrata & $0.004->4$ & $\leq 0.008$ & $\leq 0.008$ \\
Candida guilliermondii & $0.001-0.004$ & 0.002 & 0.004 \\
Candida krusei & $0.0005-0.002$ & 0.001 & 0.002 \\
Candida tropicalis & $0.00025-0.0005$ & 0.0005 & 0.0005 \\
Cryptococcus neoformans & $0.0039-0.06$ & 0.015 & 0.03 \\
Cryptococcus gattii & $0.008-0.06$ & 0.03 & 0.06 \\
Aspergillus fumigatus & $0.125-4$ & 1 & 2 \\
\hline
\end{tabular}


Each of the studies that has measured the activity of T-2307 against yeasts has used the prominent inhibition of growth as the MIC endpoint, similar to what is used for fluconazole, other azoles, and the echinocandins against yeast per the CLSI recommendations. Early studies with this investigational agent noted a trailing effect, defined as the partial inhibition of growth over an extended range of the concentrations above the MIC, when tested using the CLSI broth microdilution methods. The trailing effect observed against C. glabrata was attenuated when the glucose concentration in the growth medium was decreased to $0.1 \%$ or lower, and this effect was absent when a nonfermentative carbon source, glycerol, was used in place of glucose [14]. However, trailing was not attenuated when the $\mathrm{pH}$ of the medium was lowered. Similarly, the growth of S. cerevisiae cells was more sensitive to the inhibitory effects of furamidine or pentamidine when glycerol was the carbon source used in the growth medium rather than glucose. The clinical relevance of this is unknown, but it may influence in vitro susceptibility testing. Both the CLSI and European Union Committee on Antimicrobial Susceptibility Testing (EUCAST) antifungal susceptibility testing methods use glucose as the carbon source in their respective broth microdilution assays, rather than glycerol [24-26]. In addition, the glucose concentration in the CLSI method $(0.2 \%)$ is lower than that used by EUCAST $(2 \%)$. However, a lack of complete inhibition of growth has been observed against some isolates of different Candida species when the CLSI method has been utilized to measure the in vitro activity of this investigational agent $[12,21,22]$. It is currently unknown whether this may be strain-dependent.

T-2307 has also demonstrated an in vitro activity against some species of filamentous fungi, although the available data are limited. In a study that included several different genera of molds but with a limited number of isolates for each genus, T-2307 demonstrated activity when measured using a prominent reduction in growth as the endpoint rather than the complete inhibition of growth, as recommended by CLSI against filamentous fungi [12]. T-2307 activity was observed against Aspergillus species (MIC range: $0.0156 \mu \mathrm{g} / \mathrm{mL}$ to $4 \mu \mathrm{g} / \mathrm{mL})$, Fusarium solani $(0.125 \mu \mathrm{g} / \mathrm{mL})$, and Lichtheimia corymbifera $(0.5 \mu \mathrm{g} / \mathrm{mL})$ [12]. However, a reduction to no activity was reported against single isolates of Rhizopus oryzae (>64 $\mathrm{gg} / \mathrm{mL})$, Scedosporium boydii $(4 \mu \mathrm{g} / \mathrm{mL})$, Mucor racemosus $(2 \mu \mathrm{g} / \mathrm{mL})$, and Trichophyton rubrum $(2 \mu \mathrm{g} / \mathrm{mL})$. Fungicidal activity was limited, as the MFCs against most of the filamentous isolates ranged from $4 \mu \mathrm{g} / \mathrm{mL}$ to $>64 \mu \mathrm{g} / \mathrm{mL}$, with exceptions for the single A. nidulans $(0.0313 \mu \mathrm{g} / \mathrm{mL})$ and $A$. niger $(0.0625 \mu \mathrm{g} / \mathrm{mL})$ isolates that were included in the study.

\section{In Vivo Effectiveness}

Several studies have evaluated the in vivo effectiveness of T-2307 in experimental models of invasive candidiasis, and the in vitro activity observed for this agent has translated into efficacy against different Candida species. In a neutropenic murine model of systemic candidiasis caused by a wild-type strain of C. albicans, survival was significantly improved in a dose-dependent fashion, with $100 \%$ of mice that received doses of $0.02 \mathrm{mg} / \mathrm{kg}$ once daily surviving to the study endpoint [12]. The effective dose 50\% (ED50) calculated in this study for T-2307 was $0.00755 \mathrm{mg} / \mathrm{kg}$ when compared to $0.128 \mathrm{mg} / \mathrm{kg}$ for micafungin and $0.0466 \mathrm{mg} / \mathrm{kg}$ for amphotericin B. Similarly, in a separate study, survival was also improved in a dose-dependent fashion in immunocompetent mice with systemic candidiasis caused by an echinocandin-resistant isolate that harbored an F641S amino acid substitution in Fks1p [21]. Kidney fungal burden, as measured by colony-forming units (CFU/g), was also significantly lower in each of the T-2307 dosage groups (>3 $\log _{10}$ CFU/g lower for T-2307 at doses of $0.75,1.5,3$, and $6 \mathrm{mg} / \mathrm{kg} /$ day by subcutaneous injection) when compared to groups administered vehicle control or high-dose caspofungin $(10 \mathrm{mg} / \mathrm{kg} / \mathrm{day}$ by intraperitoneal injection). In a neutropenic murine model of invasive candidiasis caused by a C. glabrata strain harboring an Fks2p amino acid substitution (R1379S) known to result in reduced in vitro echinocandin susceptibility, the kidney fungal burden was also significantly reduced in mice treated with T-2307 at each of the above dosage levels when compared to the vehicle control and caspofungin administered at a dose of $1 \mathrm{mg} / \mathrm{kg} /$ day [20]. However, 
these reductions were less than $2 \log _{10} \mathrm{CFU} / \mathrm{g}$ and were similar to the reductions observed with high-dose caspofungin (10 mg/ $\mathrm{kg} /$ day). It is unknown if this difference in the fungal burden reduction between the $C$. albicans and C. glabrata models is due to real differences in the in vivo efficacy between these two species or due to a reduced effectiveness of T-2307 in the neutropenia setting, which was required in this model to establish the infection caused by C. glabrata. Previous work has reported differences in in vivo efficacy for anidulafungin and fluconazole between neutropenic murine and immunocompetent murine models of invasive candidiasis when the same strain of C. albicans was used to cause infection [27]. More recently, the in vivo efficacy of T-2307 has also been demonstrated against the emerging pathogen $C$. auris. In a neutropenic murine model in which infection was caused by a fluconazole-resistant isolate (fluconazole MIC $>64 \mu \mathrm{g} / \mathrm{mL}, \mathrm{T}-2307 \mathrm{MIC} \leq 0.008 \mu \mathrm{g} / \mathrm{mL}$ ), treatment with T-2307 at $3 \mathrm{mg} / \mathrm{kg} /$ day resulted both in improvements in survival and reductions in the kidney fungal burden [22]. However, no reductions in colony-forming units were observed within the brain tissue of mice treated with this investigational agent.

Interestingly, another study reported that T-2307 was effective in reducing the ocular fungal burden in mice infected with a wild-type C. albicans (strain SC5314), both when treatment was initiated shortly after intravenous inoculation (i.e., $2 \mathrm{~h}$-early-phase arm) and when delayed by $12 \mathrm{~h}$ (late-phase arm) [28]. In both the early-phase and late-phase arms, colony-forming units within the eyes and kidneys of mice treated with T-2307 at $4 \mathrm{mg} / \mathrm{kg} /$ day for three days were reduced by greater than $2 \log _{10}$ when compared to untreated mice. Reductions in the kidney and ocular fungal burden were also reported in mice treated with liposomal amphotericin B (4 mg/ $\mathrm{kg} /$ day IV) and fluconazole $(75 \mathrm{mg} / \mathrm{kg} /$ day orally) in the early-phase and late-phase arms. The ocular T-2307 trough levels were also measured $24 \mathrm{~h}$ after a single dose in both infected and uninfected mice. T-2307 concentrations between infected and uninfected mice were similar $(0.271 \mu \mathrm{g} / \mathrm{mL}$ vs. $0.245 \mu \mathrm{g} / \mathrm{mL}$, respectively), and were well above the MIC measured against the strain used to cause infection $(<0.001 \mu \mathrm{g} / \mathrm{mL})$.

T-2307 has also shown in vivo efficacy in murine models of cryptococcosis. In a neutropenic model of systemic cryptococcosis caused by C. neoformans, dose-dependent improvements in survival were reported, with survival rates of $60 \%, 70 \%$, and $100 \%$ at T-2307 doses of $0.1 \mathrm{mg} / \mathrm{kg}, 0.3 \mathrm{mg} / \mathrm{kg}$, and $1 \mathrm{mg} / \mathrm{kg}$ administered once daily, respectively, and the ED50 was calculated to be $0.117 \mathrm{mg} / \mathrm{kg}$ [12]. Against pulmonary cryptococcosis caused by C. gattii, T-2307, at a dose of $2 \mathrm{mg} / \mathrm{kg} /$ day started 2 hours postinoculation, significantly reduced the fungal burden in both the lungs and brains of mice [23]. Similar results were also noted with amphotericin B ( $1 \mathrm{mg} / \mathrm{kg} /$ day $)$ and high-dose fluconazole (160 mg/kg/day). However, only T-2307 was effective at reducing colony-forming unit counts when the start of therapy was delayed until day 14 postinfection. The authors also reported that T-2307 also prevented the progression of alveolar collapse caused by the proliferation of $C$. gattii observed within the lungs of mice not treated with this investigational agent. In vivo efficacy has also been reported against aspergillosis. In neutropenic mice infected with a wild-type $A$. fumigatus strain, survival was improved with T-2307, with survival rates of $90 \%$ at a dose of $1 \mathrm{mg} / \mathrm{kg} /$ day and $100 \%$ at a dose of $3 \mathrm{mg} / \mathrm{kg} /$ day [12].

\section{Tolerability, Safety, and Clinical Data}

Currently, no clinical data are available in the literature regarding the efficacy or safety of T-2307. A previous publication stated that this agent has successfully completed phase I studies without adverse effects [23], but these data are not currently available. In in vitro studies, as previously discussed, T-2307 has demonstrated a greater selectivity for the inhibition of yeast mitochondrial function when compared to that observed against rat and bovine mitochondria [13,19]. In addition, T-2307 has been reported to be well-tolerated in immunocompetent and neutropenic murine models of invasive fungal infections at doses of up to $6 \mathrm{mg} / \mathrm{kg} /$ day [12,14,20-23]. In contrast, pentamidine, which is structurally similar to T-2307, is known to cause numerous adverse effects/toxicities in both animals and humans, including serious endocrine/metabolic, cardiovascular, and renal toxicities 
when administered systemically [29-32]. T-2307 pharmacokinetic data are not available in the literature, and it is also unknown what formulations may be available for the treatment of patients.

\section{Conclusions}

T-2307 is an investigational agent that is currently in development for the treatment of invasive fungal infections. This agent has a novel mechanism of action and causes the collapse of the mitochondrial membrane potential, and studies have reported that this activity appears to be selective for fungi. T-2307 has both in vitro and in vivo activity against Candida species, including azole- and echinocandin-resistant strains, as well as both Cryptococcus neoformans and C. gattii. Activity has also been reported against some filamentous fungi, although the data are limited, and the complete inhibition of growth was absent or required elevated concentrations. Currently, clinical data demonstrating safety and efficacy in humans are not available.

Funding: No external funding received for the preparation of this manuscript.

Conflicts of Interest: N.P.W. has received research support from Astellas, bioMerieux, Covance, F2G, and Sfunga, and has served on an advisory board for Mayne Pharma.

\section{References}

1. WHO. Antimicrobial Resistance: Global Report on Surveillance. Available online: http://www.who.int/drugresistance/ documents/surveillancereport/en/ (accessed on 21 June 2020).

2. Alexander, B.D.; Johnson, M.D.; Pfeiffer, C.D.; Jiménez-Ortigosa, C.; Catania, J.; Booker, R.; Castanheira, M.; Messer, S.A.; Perlin, D.S.; Pfaller, M.A. Increasing Echinocandin Resistance in Candida glabrata: Clinical Failure Correlates With Presence of FKS Mutations and Elevated Minimum Inhibitory Concentrations. Clin. Infect. Dis. 2013, 56, 1724-1732. [CrossRef]

3. Vallabhaneni, S.; Cleveland, A.A.; Farley, M.M.; Harrison, L.H.; Schaffner, W.; Beldavs, Z.G.; Derado, G.; Pham, C.D.; Lockhart, S.R.; Smith, R.M. Epidemiology and Risk Factors for Echinocandin Nonsusceptible Candida glabrata Bloodstream Infections: Data From a Large Multisite Population-Based Candidemia Surveillance Program, 2008-2014. Open Forum Infect. Dis. 2015, 2, ofv163. [CrossRef]

4. Pfaller, M.A.; Castanheira, M.T.D.; Lockhart, S.R.; Ahlquist, A.M.; Messer, S.A.; Jones, R.N. Frequency of Decreased Susceptibility and Resistance to Echinocandins among Fluconazole-Resistant Bloodstream Isolates of Candida glabrata. J. Clin. Microbiol. 2012, 50, 1199-1203. [CrossRef] [PubMed]

5. Lockhart, S.R.; Etienne, K.A.; Vallabhaneni, S.; Farooqi, J.; Chowdhary, A.; Govender, N.P.; Colombo, A.L.; Calvo, B.; Cuomo, C.A.; Desjardins, C.A.; et al. Simultaneous Emergence of Multidrug-Resistant Candida auris on 3 Continents Confirmed by WholeGenome Sequencing and Epidemiological Analyses. Clin. Infect. Dis. 2017, 64, 134-140. [CrossRef] [PubMed]

6. Chow, N.A.; Gade, L.; Tsay, S.V.; Forsberg, K.; Greenko, J.A.; Southwick, K.L.; Barrett, P.M.; Kerins, J.L.; Lockhart, S.R.; Chiller, T.M.; et al. Multiple introductions and subsequent transmission of multidrug-resistant Candida auris in the USA: A molecular epidemiological survey. Lancet Infect. Dis. 2018, 18, 1377-1384. [CrossRef]

7. Chowdhary, A.; Sharma, C.; Meis, J.F. Candida auris: A rapidly emerging cause of hospital-acquired multidrug-resistant fungal infections globally. PLOS Pathog. 2017, 13, e1006290. [CrossRef]

8. Bruüggemann, R.J.M.; Alffenaar, J.C.; Blijlevens, N.M.A.; Billaud, E.M.; Kosterink, J.G.W.; Verweij, P.E.; Burger, D.M. Clinical Relevance of the Pharmacokinetic Interactions of Azole Antifungal Drugs with Other Coadministered Agents. Clin. Infect. Dis. 2009, 48, 1441-1458. [CrossRef]

9. Lewis, R.E. Current Concepts in Antifungal Pharmacology. Mayo Clin. Proc. 2011, 86, 805-817. [CrossRef]

10. Kontoyiannis, D.P.; Lewis, R.E. Treatment Principles for the Management of Mold Infections. Cold Spring Harb. Perspect. Med. 2015, 5, a019737. [CrossRef]

11. Klatt, M.E.; Eschenauer, G.A. Review of Pharmacologic Considerations in the Use of Azole Antifungals in Lung Transplant Recipients. J. Fungi 2021, 7, 76. [CrossRef] [PubMed]

12. Mitsuyama, J.; Nomura, N.; Hashimoto, K.; Yamada, E.; Nishikawa, H.; Kaeriyama, M.; Kimura, A.; Todo, Y.; Narita, H. In Vitro and In Vivo Antifungal Activities of T-2307, a Novel Arylamidine. Antimicrob. Agents Chemother. 2008, 52, 1318-1324. [CrossRef] [PubMed]

13. Shibata, T.; Takahashi, T.; Yamada, E.; Kimura, A.; Nishikawa, H.; Hayakawa, H.; Nomura, N.; Mitsuyama, J. T-2307 Causes Collapse of Mitochondrial Membrane Potential in Yeast. Antimicrob. Agents Chemother. 2012, 56, 5892-5897. [CrossRef] [PubMed]

14. Yamada, E.; Nishikawa, H.; Nomura, N.; Mitsuyama, J. T-2307 Shows Efficacy in a Murine Model of Candida glabrata Infection despite In Vitro Trailing Growth Phenomena. Antimicrob. Agents Chemother. 2010, 54, 3630-3634. [CrossRef]

15. Lanteri, C.A.; Trumpower, B.L.; Tidwell, R.R.; Meshnick, S.R. DB75, a Novel Trypanocidal Agent, Disrupts Mitochondrial Function in Saccharomyces cerevisiae. Antimicrob. Agents Chemother. 2004, 48, 3968-3974. [CrossRef] [PubMed] 
16. Nishikawa, H.; Yamada, E.; Shibata, T.; Uchihashi, S.; Fan, H.; Hayakawa, H.; Nomura, N.; Mitsuyama, J. Uptake of T-2307, a novel arylamidine, in Candida albicans. J. Antimicrob. Chemother. 2010, 65, 1681-1687. [CrossRef] [PubMed]

17. Nishikawa, H.; Sakagami, T.; Yamada, E.; Fukuda, Y.; Hayakawa, H.; Nomura, N.; Mitsuyama, J.; Miyazaki, T.; Mukae, H.; Kohno, S. T-2307, a novel arylamidine, is transported into Candida albicans by a high-affinity spermine and spermidine carrier regulated by Agp2. J. Antimicrob. Chemother. 2016, 71, 1845-1855. [CrossRef] [PubMed]

18. Toninello, A.; Via, L.D.; Siliprandi, D.; Garlid, K. Evidence that spermine, spermidine, and putrescine are transported electrophoretically in mitochondria by a specific polyamine uniporter. J. Biol. Chem. 1992, 267, 18393-18397. [CrossRef]

19. Yamashita, K.; Miyazaki, T.; Fukuda, Y.; Mitsuyama, J.; Saijo, T.; Shimamura, S.; Yamamoto, K.; Imamura, Y.; Izumikawa, K.; Yanagihara, K.; et al. The Novel Arylamidine T-2307 Selectively Disrupts Yeast Mitochondrial Function by Inhibiting Respiratory Chain Complexes. Antimicrob. Agents Chemother. 2019, 63, e00374-19. [CrossRef]

20. Wiederhold, N.P.; Najvar, L.K.; Fothergill, A.W.; Bocanegra, R.; Olivo, M.; McCarthy, D.I.; Fukuda, Y.; Mitsuyama, J.; Patterson, T.F. The novel arylamidine T-2307 demonstrates in vitro and in vivo activity against echinocandin-resistant Candida glabrata. J. Antimicrob. Chemother. 2015, 71, 692-695. [CrossRef]

21. Wiederhold, N.P.; Najvar, L.K.; Fothergill, A.W.; Bocanegra, R.; Olivo, M.; McCarthy, D.I.; Kirkpatrick, W.R.; Fukuda, Y.; Mitsuyama, J.; Patterson, T.F. The Novel Arylamidine T-2307 MaintainsIn Vitroand In Vivo Activity against EchinocandinResistant Candida albicans. Antimicrob. Agents Chemother. 2014, 59, 1341-1343. [CrossRef]

22. Wiederhold, N.P.; Najvar, L.K.; Jaramillo, R.; Olivo, M.; Patterson, H.; Connell, A.; Fukuda, Y.; Mitsuyama, J.; Catano, G.; Patterson, T.F. The Novel Arylamidine T-2307 Demonstrates In Vitro and In Vivo Activity against Candida auris. Antimicrob. Agents Chemother. 2019, 64. [CrossRef]

23. Nishikawa, H.; Fukuda, Y.; Mitsuyama, J.; Tashiro, M.; Tanaka, A.; Takazono, T.; Saijo, T.; Yamamoto, K.; Nakamura, S.; Imamura, Y.; et al. In vitro and in vivo antifungal activities of T-2307, a novel arylamidine, against Cryptococcus gattii: An emerging fungal pathogen. J. Antimicrob. Chemother. 2017, 72, 1709-1713. [CrossRef] [PubMed]

24. CLSI. Reference Method for Broth Dilution Antifungal Susceptibility Testing of Filamentous Fungi, Document M38, 3rd ed.; Clinical and Laboratory Standards Institute: Wayne, PA, USA, 2017.

25. CLSI. Reference Method for Broth Dilution Antifungal Susceptibility Testing of Yeasts, Document M27, 4th ed.; Clinical and Laboratory Standards Institute: Wayne, PA, USA, 2017.

26. Arendrup, M.C.; Cuenca-Estrella, M.; Lass-Flörl, C.; Hope, W. EUCAST technical note on the EUCAST definitive document EDef 7.2: Method for the determination of broth dilution minimum inhibitory concentrations of antifungal agents for yeasts EDef 7.2 (EUCAST-AFST)*. Clin. Microbiol. Infect. 2012, 18, E246-E247. [CrossRef] [PubMed]

27. Wiederhold, N.P.; Najvar, L.; Bocanegra, R.; Kirkpatrick, W.; Patterson, T. Comparison of anidulafungin's and fluconazole's in vivo activity in neutropenic and non-neutropenic models of invasive candidiasis. Clin. Microbiol. Infect. 2012, 18, E20-E23. [CrossRef]

28. Abe, M.; Nakamura, S.; Kinjo, Y.; Masuyama, Y.; Mitsuyama, J.; Kaku, M.; Miyazaki, Y. Efficacy of T-2307, a novel arylamidine, against ocular complications of disseminated candidiasis in mice. J. Antimicrob. Chemother. 2019, 74, 1327-1332. [CrossRef] [PubMed]

29. Coyle, P.; Carr, A.D.; Depczynski, B.B.; Chisholm, D.J. Diabetes mellitus associated with pentamidine use in HIV-infected patients. Med. J. Aust. 1996, 165, 587-588. [CrossRef] [PubMed]

30. Lionakis, M.S.; Chamilos, G.; Lewis, R.E.; Wiederhold, N.P.; Raad, I.I.; Samonis, G.; Kontoyiannis, D.P. Pentamidine Is Active in a Neutropenic Murine Model of Acute Invasive Pulmonary Fusariosis. Antimicrob. Agents Chemother. 2006, 50, 294-297. [CrossRef] [PubMed]

31. O’Brien, J.G.; Dong, B.J.; Coleman, R.L.; Gee, L.; Balano, K.B. A 5-Year Retrospective Review of Adverse Drug Reactions and Their Risk Factors in Human Immunodeficiency Virus-Infected Patients Who Were Receiving Intravenous Pentamidine Therapy for Pneumocystis carinii Pneumonia. Clin. Infect. Dis. 1997, 24, 854-859. [CrossRef]

32. Helmick, C.G.; Green, J.K. Pentamidine-Associated Hypotension and Route of Administration. Ann. Intern. Med. 1985, $103,480$. [CrossRef] 